[6] Shipley Photo Resist, Technical Manual, Jan. 1978.

[7] J. S. T. Huang and G. W. Taylor, "Modeling of an ion-implanted silicon-gate depletion-mode IGFET," IEEE Trans. Electron Devices, vol. ED-22, pp. 995-1001, Nov. 1975.

\section{A General Model for the Frequency Response of Multiphase Charge Transfer Delay Lines}

\section{HANS WALLINGA}

\begin{abstract}
A general model for the transfer function of a multiphase CTD delay line is presented in the $z$-domain. It covers the widely used single-phase transfer function. The new model has been applied for CTD's with asymmetrical potential wells. If poor charge transfer efficiencies are considered, the new model offers a significant correction to the single phase model.
\end{abstract}

The widely used linearized small-signal expression for the transfer function of a charge transfer device delay line in the $z$-transform notation [1]-[3] is

$$
H(z)=\left(\frac{1-p \epsilon}{1-p \epsilon z^{-1}}\right)^{N} z^{-N} \text {. }
$$

Here $p$ is the number of transfer phases, $N$ is the number of delay elements (each built up of $p$ transfer stages), and $\epsilon$ is the small-signal charge transfer inefficiency of one transfer stage. The frequency response is obtained by substitution of $z=$ $\exp (j \omega T)$ into (1). $T$ denotes the clock period and is equal to the sampling time interval; $\omega$ is the circular signal frequency. The resulting well-known expressions for the modulus and extra phase shift of the CTD frequency response are, respectively,

$$
|H(j \omega T)|=\left(\frac{(1-p \epsilon)^{2}}{1-2 p \epsilon \cos \omega T+p^{2} \epsilon^{2}}\right)^{N / 2}
$$

and

$$
\Delta \Phi(\omega T)=N \arctan \left(\frac{p \epsilon \sin \omega T}{1-p \epsilon \cos \omega T}\right) .
$$

Equation (1) has essentially been derived from a single transfer per delay element concept. In order to cover multiphase delay elements, consisting of $p$ transfer stages, they have been modeled as single transfer stages with transfer inefficiency $p \epsilon$.

In practice, unidirectional charge transport in a CTD requires multiphase delay elements. This correspondence deals with a general linearized small-signal model for the transfer function of one single transfer stage of a $p$-phase CTD. The extension with respect to the single-phase model is that it includes the composition of new signal charge packets out of transferred and remaining parts at each of the $p$ storage locations in a $p$-phase delay element instead of only at the end of a delay element. The overall frequency response of a $p$-phase CTD delay line of $N$ delay elements is then modeled by cascading

Manuscript received March 1, 1978; revised July 25, 1978. This research was partly prepared while the author was on a fellowship at the CNR-LAMEL, Bologna, Italy. The fellowship was granted by the Consiglio Nazionale delle Ricerche, Italy, on the proposal of the Nether" lands Organization for the Advancement of Pure Research (Z.W.O.).

The author is with the Department of Electrical Engineering, Twente University of Technology, Enschede, The Netherlands. $p N$ single transfer stages. First, a few definitions are given and the basic assumptions are discussed briefly.

An important requirement for the validity of the model to be presented is that the system behaves linearly. To meet this, the system has been linearized by considering only small-signal charge packets $q$ as a modulation on the background charge packets $Q_{b}$ [4]. This background charge, frequently referred to as "fat zero charge," is not a function of frequency and it has to be considered as a dc biasing parameter. The charge transfer of $Q_{b}$ is therefore omitted in the following discussion. Charge loss to or from the substrate is neglected.

The CTD delay line is a discrete time system, handling charge samples of an electrical input signal. In consequence, the charge packets usually are only defined at integer multiples of the sampling interval $T$ (apart from a constant delay of the input stage). Because in a $p$-phase CTD the charge samples are subjected to $p$ transfers within one period $T$, the charge samples can physically also be identified it integer multiples of the delay time $T_{1}$ per transfer stage, with $T_{1}=T / p$. Consider the $n$th delay element in a $p$-phase CTD. It has been modeled as a sequence of $p$ storage locations $C_{n, j},(1 \leqslant i \leqslant p)$, which are connected by transfer stages. The charge $q_{n, j}$ stored at $C_{n, j}$ has to be defined at the time sequences $\left(k T+j T_{1}\right)$. The transfer of $q_{n, j}$ towards $C_{n, j+1}$ takes place in the time interval $\left(k T+j T_{1}\right)<t<\left(k T+(j+1) T_{1}\right)$.

Physically, the small-signal charge packet $q_{n, j}$ consists, for the greater part, of free charge carriers, but a minor part may be trapped in interface or bulk states. It is assumed that in $C_{n, j}$ at time sequences $k T+j T_{1}$, those states are occupied according to equilibrium with the total charge $Q_{b}+q_{n, j}\left(k T+j T_{1}\right)$ in the charge packet [5]. The signal charge that has been trapped is immobile as long as it has not been emitted. After the charge packet has been transferred, a part $\epsilon$ of $q_{n, j}$ remains at the storage location $C_{n, j}$.

Now it is very likely that the occupancy of the interface and bulk states is not in equilibrium with the free charge carrier density, and in the period before the next charge packet arrives, emission of signal charge from those states is conceivable $[5],[6]$. At the arrival of the next charge packet, a part of the originally trapped signal charge plus a part of the remaining free signal charge may still reside at $C_{n, j}$. The residing part is defined as $\epsilon_{0}$. The part of $q_{n, j}$ that flows backwards $i$ $(1 \leqslant i \leqslant p-1)$ storage locations before it is added to the next charge packet is defined as $\epsilon_{i}$. It is assumed that no forward flow of the remaining charge occurs after the actual transfer of the signal charge packet finished. Including $\epsilon_{0}$ it follows

$$
\sum_{i=0}^{p-1} \epsilon_{i}=\epsilon
$$

For the sake of clearness and simplicity, it is further assumed that the sequential transfer stages behave equally. The discussion above can be summarized in the following equation:

$$
\begin{aligned}
q_{n, j}\left(k T+j T_{1}\right)= & (1-\epsilon) q_{n, j-1}\left(k T+(j-1) T_{1}\right) \\
& +\sum_{i=0}^{p-1} \epsilon_{i} q_{n, j+i}\left((k-1) T+(j+i) T_{1}\right)
\end{aligned}
$$

After $z$-transformation this gives

$$
q_{n, j}(z)=(1-\epsilon) z^{-1 / p} q_{n, j-1}(z)+z^{-1} \sum_{i=0}^{p-1} \epsilon_{i} z^{i / p} q_{n, j+i}(z) .
$$




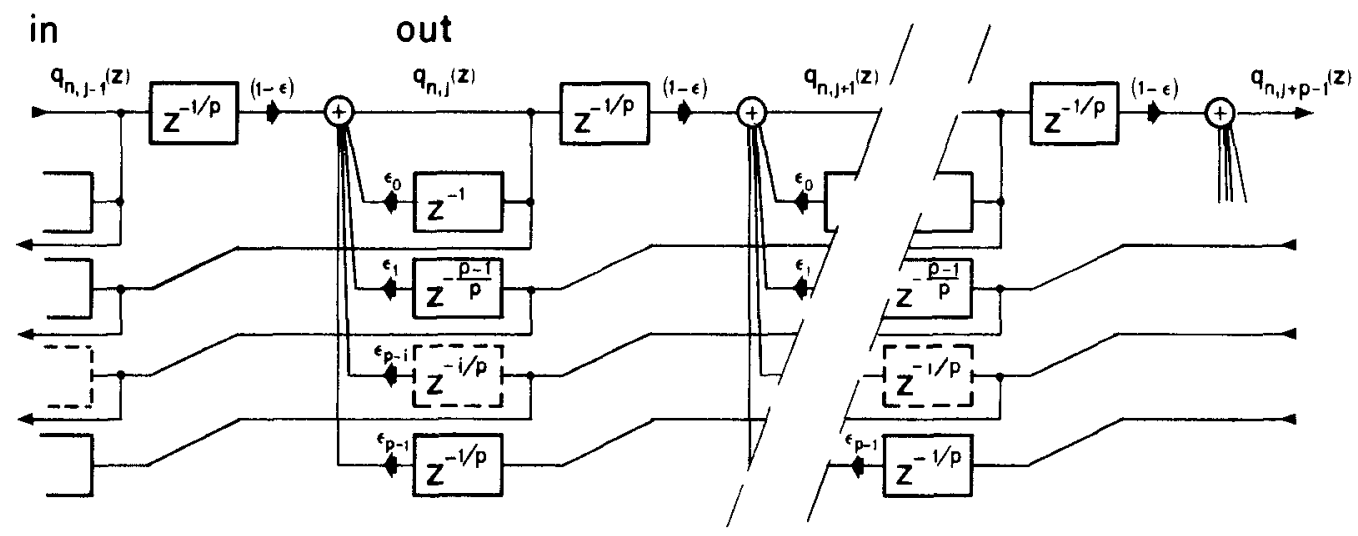

Fig. 1. General $z$-transform model of a transfer stage of a $p$-phase CTD.

In Fig. 1 a schematic representation of this equation is shown. From (6) follows the equation for the transfer function $H(z)$ :

$$
H(z)=(1-\epsilon) z^{-1 / p}+z^{-1} \sum_{i=0}^{p-1} \epsilon_{i} z^{i / p} H(z)^{i+1}
$$

For $p>2$, generally no useful analytical solution of (7) is obtained. Under appropriate assumptions, however, several simplified models may be deduced. For $p=1$, the single transfer model applied in (1) is obtained.

As an illustration, a model for CTD's in which no backward flow of residual charge occurs is discussed in the following. Physically, the assumption of absence of backward flow is quite acceptable for CTD's with asymmetric potential wells. Asymmetric potential wells are essentially required in twophase and semi-two-phase devices. Most of the applied asymmetric potential wells are obtained by barrier implant or stepped oxide. They form a local potential well when the next clock pulse is no longer applied, i.e., after the transfer has finished. This local well collects all the residing charge from this transfer and prevents any backward flow of charge. For three- and four-phase devices, a model without backflow of charge probably may be applied if the remaining signal charge mainly has been trapped in interface states. The above model is characterized by $\epsilon=\epsilon_{0}$ and $\epsilon_{i}=0$ for $i \geqslant 1$. Equation (7) now simplifies to

$$
H_{1}(z)=\frac{(1-\epsilon) z^{-1 / p}}{1-\epsilon z^{-1}}
$$

The associated schematic structure is shown in Fig. 2. The overall transfer function of the CTD delay line is obtained by cascading $p N$ transfer stages:

$$
H(z)=\left(\frac{1-\epsilon}{1-\epsilon z^{-1}}\right)^{p N} z^{-N}
$$

The modulus of the frequency response is now

$$
|H(j \omega T)|=\left(\frac{(1-\epsilon)^{2}}{1-2 \epsilon \cos \omega T+\epsilon^{2}}\right)^{p N / 2} .
$$

The extra phase shift due to charge transfer inefficiency is

$$
\Delta \Phi(\omega T)=p N \arctan \left(\frac{\epsilon \sin \omega T}{1-\epsilon \cos \omega T}\right) .
$$

In Fig. 3 the relative errors of the modulus and extra phase shift of the frequency response obtained by using (2) and (3)

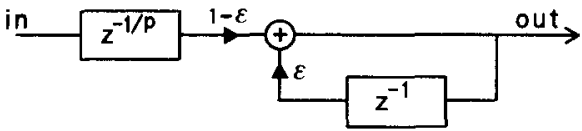

Fig. 2. $z$-transform model of a transfer stage of a $p$-phase CTD in the absence of backflow of residual charge.

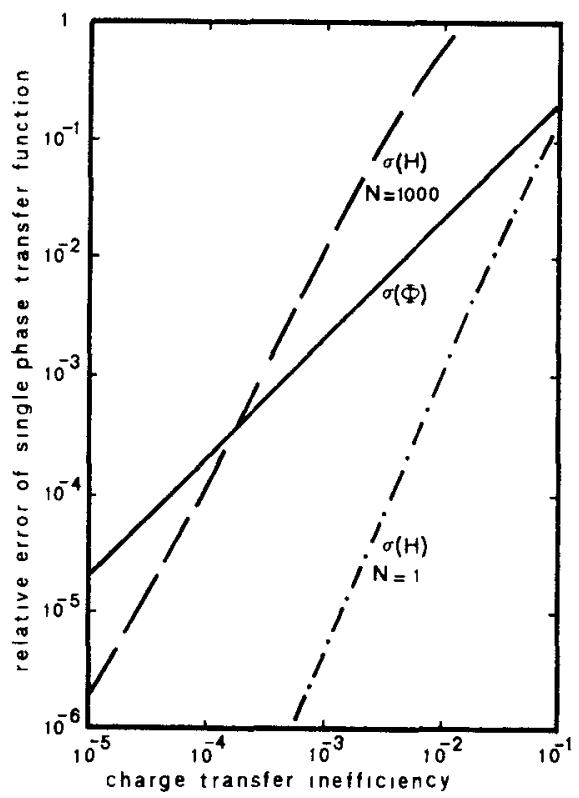

Fig. 3. Relative error $\sigma(H)$ of modulus and $\sigma(\phi)$ of extra phase shift, if the single phase transfer function expressions (3) and (4) are used instead of the multiphase expressions (7) and (8), versus the charge transfer inefficiency $\epsilon ; p=4$. $-\sigma(H)$ for $N=1000, \omega T=\pi / 2$. $-\cdot \ldots \ldots \sigma(H)$ for $N=1, \omega T=\pi / 2$. $\square \sigma(\phi), \omega T=\pi / 4$, independent of $N$.

instead of (10) and (11) are shown as a function of $\epsilon$ for a four-phase 1000-element CTD and for one four-phase delay element. For uniform and small charge transfer inefficiencies, as they hold for representative modern CTD's, (1), (2), and (3) can be seen as an admissible approximation, and the improvement obtained by using (9), (10), and (11) has little practical meaning. It is, however, well known that for small background charge packets, the charge transfer inefficiency increases [7]-[9], and for research in this field, the presented 
model will be useful. The basic $z$-transform model for one transfer stage [Fig. 2 and (8)] also directly applies for the description of multiplexed multiphase CTD delay lines [10], [11].

\section{CONCLUSION}

A linearized model describing the effect of incomplete charge transfer on the transfer function of a $p$-phase CTD has been presented. The model covers the widely used singlephase expressions. For CTD's with asymmetrical potential well, it has been shown that the presented model predicts negligible deviations from the single transfer model, as long as small inefficiencies $\left(\epsilon<10^{-3}\right)$ are considered, but for larger $\epsilon$ values, considerable corrections are apparent. The new multiphase model is therefore particularly suited for research concerning the transfer inefficiency for small background charge packets because then large $\epsilon$ may be expected. In the case of strongly nonuniform $\epsilon$ of the different transfer stages, large $\epsilon$ values may occur, and the presented model will prove to be very useful. Besides, the presented model is founded on a better theoretical basis, and under appropriate assumptions several simplified models may be deduced.

\section{ACKNOWLEDGMENT}

The author wishes to thank O. W. Memelink for his current interest in this work, and D. D. Buss for his valuable review comments.

\section{REFERENCES}

[1] D. D. Buss and W. H. Bailey, "Applications of charge transfer devices to communication," in Proc. Conf. on Applications of CCD 's, San Diego, CA, Sept. 1973, pp. 83-93.

[2] G. F. Vanstone, J. B. G. Roberts, and A. E. Long, "The measurement of the charge residual for CCD transfer using impulse and frequency responses," Solid-State Electron., vol. 17, pp. 889895 , Sept. 1974

[3] C. H. Séquin and M. F. Tompsett, "Charge transfer devices," in Advances in Electronics and Electron Physics, suppl. 8. New York: Academic, 1975.

[4] C. N. Berglund, "Analog performance limitations of chargetransfer dynamic shift registers," IEEE J. Solid-State Circuits, vol. SC-6, pp. 391-394, Dec. 1971.

[5] J. E. Carnes and W. F. Kosonocky, "Fast-interface-state losses in charge-coupled devices," Appl. Phys. Lett., vol. 20, pp. 261-263, Apr. 1972.

[6] D. L. Heald, "Theoretical analysis of charge transfer loss arising from interface states in charge-coupled devices," Solid-State Electron., vol. 20, pp. 657-663, Aug. 1977.

[7] W. F. Kosonocky and J. E. Carnes, "Two phase charge coupled devices with overlapping poly-silicon and aluminum gates," $R C A$ Rev., vol. 34, pp. 164-202, Mar. 1973.

[8] W. J. Bertram, A. M. Mohsen, F. J. Morris, D. A. Sealer, C. H. Séquin, and M. F. Tompsett, "A three-level metallization threephase CCD," IEEE Trans. Electron Devices, vol. ED-21, pp. 758 767 , Dec. 1974.

[9] R. W. Broderson, D. D. Buss, and A. F. Tasch, Jr., "Experimental characterization of transfer efficiency in charge coupled devices," IEEE Trans. Electron Devices, vol. ED-22, pp. 40-46, Feb. 1975.

[10] J. M. Caywood and D. D. Buss, "Frequency response of a multiplexed charge transfer delay line," IEEE J. Solid-State Circuits, vol. SC-9, pp. 310-311, Oct. 1974.

[11] S. C. Dutta Roy, "Frequency response of a multiplexed charge transfer delay line," Electron. Lett., vol. 12, pp. 280-281, May 1976; “Errata,” p. 391, July 1976.
A CMOS Bandgap Voltage Reference

\author{
G. TZANATEAS, C. A. T. SALAMA, AND Y. P. TSIVIDIS
}

Abstract-A simple micropower CMOS bandgap voltage reference is described. The reference utilizes MOS devices operating in the weak inversion region in conjunction with a process compatible bipolar device. The voltage reference is insensitive to threshold and mobility variations and is independent of the slope factor which characterizes weak inversion.

\section{INTRODUCTION}

The design of CMOS single chip analog-to-digital conversion systems would be considerably simplified by the implementation of a reference voltage generated on-chip. A CMOS reference voltage source utilizing devices operated in weak inversion has been recently described [1]. In principle, the reference uses a pair of MOSFET's to generate a differential voltage with a positive temperature coefficient which is canceled out by using the negative temperature coefficient of a forward-biased diode connected transistor. The output voltage of that reference is dependent on the slope factor $n$ which characterizes weak inversion operation [2]. Because of the strong temperature coefficient of $n$, the temperature coefficient of the output voltage cannot be made as small as is required in several precision analog/digital applications.

The object of this correspondence is to describe an improved voltage reference source utilizing devices operating in the weak inversion region. The voltage reference is insensitive to threshold and mobility variations, is independent of the factor $n$, and is compatible with CMOS technology.

\section{Circuit Realization}

A simple model [2] can be used to describe the operation of an $\mathrm{n}$-channel MOS transistor in weak inversion. The model assumes that: 1) the channel is sufficiently long so that the gradual channel approximation can be used and channel length modulation effects are negligible; 2) generation currents in the space-charge regions associated with the source, drain, and gate regions are negligible; and 3) that the density of surface states and the fluctuations of surface potential are negligible. Under these conditions the $I-V$ characteristics of an $n$-channel MOST can be described by the following equation [2]:

$$
I_{D} \simeq I_{D O} S e^{q V_{G / n k T}}\left(e^{-q V_{S / k T}}-e^{-q V_{D / k T}}\right)
$$

where $I_{D O}$ is a characteristic current; $n$ is a slope factor; $S$ is the geometrical shape factor of the transistor (effective width over effective length of the channel); $V_{S}, V_{D}$, and $V_{G}$ are the source to substrate, drain to substrate, and gate to substrate voltages, respectively. The slope factor $n$ may be considered as a constant for transistors biased by similar values of $V_{S}$. The same is true for the characteristic current $I_{D O}$ for transistors on the same chip. $I_{D O}$ however is very poorly controlled from batch to batch as an absolute value.

The upper limit of validity of (1) to ensure weak inversion operation under saturated drain condition $\left(V_{D}-V_{S}>3 \mathrm{kT} / q\right)$

Manuscript received June 15, 1978; revised September 6, 1978. This work was supported by the National Research Council of Canada.

G. Tzanateas and C. A. T. Salama are with the University of Toronto, Toronto, Ont., Canada.

Y. P. Tsividis is with Columbia University, New York, NY 10027. 